\title{
Análise da distribuição dos fatores de risco para internações repetidas em idosos residentes em área rural
}

\author{
Analysis of the distribution of the risk factors for repeated \\ hospitalization of elderly patients resident in the countryside.
}

\section{Resumo}

Fabiana Breitenbach Luis Antốnio Benvegnú2 Edilểia Fischer

O envelhecimento da população e seus desdobramentos na área da saúde são um desafio para os profissionais que atuam em Atenção Primária à Saúde. A mudança da assistência e a sua organização baseada em informações sobre as condições de saúde desta população e seus determinantes são fundamentais. Este artigo analisa a distribuição da população idosa rural quanto a seis fatores de risco para internações repetidas, são eles: doenças crònicas, número de consultas, faixa etária, presença ou não de apoio social e autopercepção da saúde. Utilizaram-se dados secundátios provenientes de informações existentes nos prontuários de 176 idosos cadastrados em um PSF localizado em área rural do município de Santa Rosa (RS). Na análise dos dados, evidenciou-se que a idade média era de 65-75 anos de idade, composta, em sua maioria, de mulheres, e a autopercepção de saúde foi considerada média por $63,6 \%$ dos analisados. $\mathrm{O}$ objetivo deste estudo foi o de conhecer a população idosa rural da ESF para um melhor planejamento das ações propostas a essa população. Novas concepções de assistência à saúde dos idosos devem ser agregadas as ESF, com a utilização de novas tecnologias voltadas para a qualificação da asistência ao idoso.

\begin{abstract}
Aging of the population and its developments in the bealth area are a challenge to the professionals who act in Primary Health Care. The change in assistance and its organization based on information about the bealth conditions of this population and its determining factors are essencial. This article analyzes the distribution of rural elderly population as to six risk factors for repeated bospitalizations. They are: chronio diseases, number of visits, age group, existence or not of social support and self-perception of bealth. Secondary data derived from information existing in the charts of 176 registered elderly patients in a Family Health Program located in a rural area of the municipality of Santa Rosa (RS) were used. In the data analysis, it was observed that the average age was 65-75 years, consisting mostly of nomen; the self-perreption of bealth was considered average by $63.6 \%$ of the people analyzed. The abjective of this study was to know the rural elderly population of Family Health Strategy for better planning of the proposed actions to this population. New conceptions of bealth assistance to the elderly must be added to the Family Health Strategy, with the use of new technologies turned to the qualification of the assistance to the elderly.
\end{abstract}

Palavras-chave: Saúde do idoso; Hospitalização; Fatores de Risco; Programa Saúde da Família; População Rural.
Key Words: Health for the Elderly; Hospitalization; Risk Factor; Fannily Health Program; Rural Population.

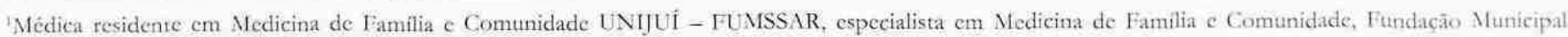
de Saude, Santa Rosa, Rio Grande do Sul, Brasil.

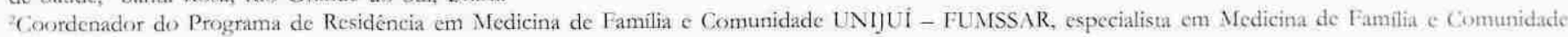
- SBMIFC, Rio Grande do Sul, Brasil.

Preceptora do Programa de Residéncia em Medicina de Familia e Comunidade UNIJUI - FUMSSMR, médiea bomeopara, Rio Grande do Sul, Brasil. 


\section{Introdução}

A OMS estima que existirão 1,2 bilhões de pessoas com mais de 60 anos no ano de 2025 e 2 bilhões em 2050. Atualmente, dois terços da população idosa vivem em países em desenvolvimento; em 2025 serão $75 \%{ }^{1}$. Juntos, esses países somam $62 \%$ da população idosa mundial. O Brasil pertence ao grupo de 10 países com aumento da população de pessoas de mais de 60 anos de idade ou mais em termos absolutos. Com esse aumento, o país, no ano de 2025, estará com a sexta população de idosos do mundo. A população brasileira vem envelhecendo de forma rápida desde a década de $1960 \mathrm{com}$ a queda das taxas de fecundidade ${ }^{2}$.

O envelhecimento populacional tem como principal desencadeante a redução da mortalidade por causas infecciosas, processo este denominado transição demográfica.

A transição epidemiológica refere-se às modificações a longo prazo dos padrões de morbidade, invalidez e morte, as quais caracterizam uma população específica, e que, em geral, ocorrem em conjunto com outras transformações demográficas, sociais e econômicas. No Brasil, esse processo inicia-se na década de 1940, quando as mortes por doenças infecciosas tiveram uma queda drástica ${ }^{3}$. No entanto apresenta características distintas de países desenvolvidos ou mesmo de países vizinhos. Há uma superposição entre as etapas, em que convivem doenças transmissíveis (com a reintrodução de doenças como malária, hanseníase) e crònico-degenerativas, a morbi-mortalidade persiste em ambos os padrões, caracterizando uma transição prolonga$\mathrm{da} \mathrm{a}^{4}$.

De acordo com a Pesquisa Nacional por Amostra de Domicilio, realizada em 2003, a população de idosos representa um contingente de quase 17,6 milhões de pessoas com 60 anos ou mais $(9,7 \%$ da população brasileira). As mulheres são maioria, 8,9 milhões $(62,4 \%)$ dos idosos são responsáveis pelos domicílios e têm, em média, 69 anos de idade e 3,4 anos de estudo. $O$ índice de envelhecimento passou de 0,1 no início da década de 1980 para 0,25 em 2004, ou seja, para cada 100 jovens existem 25 idosos 5 .

No Rio Grande do Sul, os idosos (+ de 60 anos de idade) representam $12 \%$ da população, totalizando 1.285.882 habitantes, percentual acima do verificado no Brasil.

Ao longo do século XX ampliou-se muito a expectativa de vida dos brasileiros. A das mulheres praticamente dobrou entre 1910 (quando era de 34,6 anos) e 1990 (quando passou a 69,1). A expectativa de vida masculina cresceu 28,9 anos no período, passando de 33,4 anos em 1910 para 62,3 anos em 1990. A tendência de aumento se manteve até o fim do século XX. Em 2000, a expectativa de vida para ambos os sexos era de 68,6 anos $(64,8$ para homens e 72,6 para mulheres) ${ }^{6}$. Em Santa Rosa, cidade onde foi realizado o estudo, a expectativa de vida, que era de 69,2 anos no triênio 1980-1982 aumentou para 72,7 em 2000-20047.

O crescimento da população idosa leva, inevitavelmente, a um aumento dos recursos dispendidos na área de saúde. As doenças próprias do envelhecimento ganham uma maior expressão no conjunto da sociedade aumentando a demanda dos serviços de saúde. As enfermidades são, em geral, crônicas, exigindo acompanhamento por longos períodos, e as internações hospitalares são mais frequentes.

Dados do IBGE mostram que $69 \%$ dos idosos brasileiros relatam ter pelo menos uma doença crônica. As doenças relatadas com mais frequência são hipertensão, artrites, doenças do coração, diabetes, asma, doença renal crônica, câncer e cirrose (por ordem decrescente de frequência) $)^{5}$. Tal realidade tem implicações sérias no que se refere ao complexo de problemas de saúde do país, pois a redução da mortalidade sem melhoria da qualidade de vida, acaba por incrementar a morbidade. As necessidades em saúde apresentam um padrão de distribuição, em "J", segundo a idade, ou seja, as pessoas no inicío e no final da vida apresentam mais problemas de saúde ${ }^{4}$.

Alguns indicadores podem auxiliar no conhecimento das condições de sáude de uma determinada população. Tais como: perfil de morbi-mortalidade, uso de serviços de saúde e fatores de risco 4 .

A utilização dos serviços de saúde, dados colhidos 
em 2003 na PNAD, é maior nas faixas etárias dos extremos ${ }^{5}$. No entanto, ainda este acesso apresenta-se de modo desigual infuenciado pelo nível socioeconômico do idoso.

Quanto às internações, 12,3 milhòes de pessoas internaram-se no ano anterior ao da pesquisa ( 7 para cada 100 habitantes) sendo que a faixa de 65 anos ou mais teve um coeficiente de internação de 14 por 100 habitantes 5 . No Japão, $10 \%$, 14\% no Reino Unido, $16 \%$ na Alemanha e $18 \%$ nos EUA e Canadá. As internações são mais frequentes em faixas socioeconómicas mais baixas. No Brasil, os custos com as internações de câncer, doenças cardiovasculares e diabetes, na rede hospitalar contratada do Inamps, atingiram, no ano de 1985, 135 milhões de dólares, significando mais de $1 / 3$ dos custos de todas as internações. As visitas ao médico no Brasil estão dentro das variações internacionais ( $28 \%$ não haviam visitado).

Com vistas nesta realidade, em dezembro de 1999 , cria-se a Política Nacional de Saúde do Idoso, a qual, em sua introdução, assume que o principal problema que pode afetar o idoso, como consequência da evolução de suas enfermidades e de seu estilo de vida, é a perda de sua capacidade funcional. Traz em seu texto a promoção de envelhecimento saudável, a manutenção e a melhoria, ao máximo, da capacidade funcional dos idosos, a prevenção de doenças, a recuperação da saúde dos que adoecem e a reabilitação daqueles que venham a ter a sua capacidade funcional restringida, de modo a garantir-lhes permanência no meio em que vivem, exercendo, de forma independente, suas funções na sociedade'.

O envelhecimento populacional constitui, portanto, um desafio para a saúde pública atual, pois esta terá de cuidar de uma grande população idosa, com baixa renda e uma alta prevaléncia de doenças crônicas.

Para o alcance do propósito da Política Nacional de Saúde do Idoso, foram definidas como diretrizes essenciais a promoção do envelhecimento saudável; a manutenção da capacidade funcional; $a$ assistência às necessidades de saúde do idoso; a reabilitação da capacidade funcional comprometida; a capacitação de recursos humanos especializados; $\triangleright$ apoio a desenvolvimento de cuidados informais; e o apoio a estudos e pesquisas.

Assim, o grande desafio para o sistema de saude é conseguir traduzir os avanços obtidos no campo legal em mudanças efetivas e resolutivas da prática da atenção à saúde da populaçào. O êxito da reforma proposta com o uso potencializado da atenção básica, complementada pela rede de serviços especializados e hospitalares, tem sido a busca permanente dos gestores de saúde.

A Estratégia da Família, criado em 1994, aparece como a principal política pública para dar resposta ao complexo atendimento ao idoso ${ }^{\$}$. Tem como diretrizes: reconhecer a saúde como um direito de cidadania, humanizando as práticas de saúde e buscando a satisfaça do usuário pelo seu estreito relacionamento com os profissionais de saúde; prestar assistência universal, integral, equánime, contínua e, acima de tudo, resolutiva e de boa qualidade à população, na unidade de saúde e no domicílio, elegendo a família, em seu contexto social, como núcleo básico de abordagem no atendimento à saúde; identificar os fatores de risco aos quais a população está exposta e neles intervir de forma apropriada; proporcionar o estabelecimento de parcerias pelo desenvolvimento de açòes intersetoriais que visem à manutençào e à recuperaçào da saúde da população; estimular a organizaçào da comunidade para o efetivo exercício do controle social.

Segundo Chaimowicz ${ }^{2}$, há duas linhas de açào que minimizariam o impacto do envelhecimento populacional sobre os serviços de saúde: o incremento ao apoio formal e informal ao idoso e a compressão da morbidade que se refere ao tempo entre o início das doenças ou incapacidades e a morte.

Os trabalhadores em saúde, inseridos nesse contexto, devem ter uma visão integral do idoso e de sua familia, trabalhando com suas reais necessidades e disponibilidades, valendo-se de uma prática tecnicamente competente e humanizada, pelas ações de promoção, proteção e recuperação da saúde. A promoção da saúde é considerada uma alternativa com melhor custo-beneficio para que ocorra um envelhecer menos incapacitante, devendo ser enfatizada jả nos cursos de graduaçào e na formaçào de profissionais 
que atuam na Atenção Primária à Saúde².

O objetivo deste estudo foi analisar a distribuição dos principais fatores de risco para internações hospitalares repetidas em idosos cadastrados em uma Unidade da Estratégia de Saúde da Família (ESF) da zona rural de Santa Rosa (RS).

\section{Material e Métodos}

Tipo de Estudo e população alvo: foi realizado um estudo epidemiológico do tipo transversal. A população-alvo foi composta por idosos cadastrados pela ESF, residentes no distrito rural de Bela União. Foram analisados, nos meses de janeiro a março de 2006, 176 prontuários, 0 que correspondia a $83,8 \%$ da população idosa da área.

Utilizou-se o conceito da OMS que julga idosa a população acima de 60 anos de idade em países em desenvolvimento. Da mesma forma, no Brasil, a Lei 8.842 estabelece que individuos com 60 anos de idade ou mais sejam considerados idosos?.

\section{Local do Estudo}

O municipio de Santa Rosa está localizado na região noroeste do Estado do Rio Grande do Sul, com uma população estimada em 69.989 habitantes, $9.743(13,9 \%)$ em área rural ${ }^{5}$. A principal atividade econòmica é a agricultura, destacando-se o plantio de soja, milho e trigo.

A Fundação Municipal de Saúde, órgão da administração direta da Prefeitura Municipal de Santa Rosa, organizou os serviços de saúde em Distrios Sanitảrios (onze em área urbana e dois em área rural). Cada um desses distritos tem área de abrangência definida e população adscrita.

$\mathrm{O}$ distrito de saúde Bela União localiza-se na área rural do munícipio, há $20 \mathrm{~km}$ da sede do município e consta com uma população aproximada de 3.000 pessoas. A unidade de saúde onde foi realizado o estudo tem como trabalhadores em saúde uma ESF composta de um médico de familia e comunidade, um enfermeiro, dois técnicos de enfermagem, um dentista com pós-graduação em Saúde Pública, um acidente em consultório dentário e seis agentes de saúde, todos em regime de 40 horas semanais com de- dicação exclusiva.

\section{Coleta dos Dados}

O prontuário de cada idoso continha um questionário baseado nos fatores de risco para internações repetidas descritos por Boult et al e validado por Veras ${ }^{10}$, para a população brasileira. $\mathrm{O}$ instrumento é composto de oito perguntas que traduzem fatores de risco para internações repetidas (idade, sexo, disponibilidade de cuidador, autopercepção da saúde, presença de doença cardíaca, presença de diabetes mellitus, pernoite hospitalar, consulta médica nos últimos 12 meses). Os dados utilizados neste estudo foram obtidos a partir desses questionários.

\section{Variáveis}

Para estabelecer a idade, utilizou-se a data de nascimento e agrupou-se em cinco faixas etárias (60-64 anos, 65-74 anos, 75-79 anos, 80-84 anos, e acima ou igual a 85 anos).

A disponibilidade de cuidador foi avaliada com a pergunta: "existe algum amigo, parente ou vizinho que poderia tomar conta de você por alguns dias se você precisar?" Com duas respostas possíveis: sim e não.

A autopercepção de saúde contempla aspectos da saúde física, cognitiva e emocional e tem se mostrado um método consistente e robusto para aferir as condições de saúde de uma população ${ }^{11}$. Está associada ao uso dos serviços de saúde e à mortalidade. Foi utilizada a pergunta: "como o entrevistado considera a sua saúde?", tendo como respostas: excelente, muito boa, boa, média e ruim.

A presença de doença cardíaca foi avaliada com a pergunta: "você alguma vez já teve doença coronariana? Angina? Infarto do miocárdio? Ataque do coração?" Com alternativas sim/não para cada questão. A presença de doença cardíaca era considerada positiva se qualquer uma das respostas fosse sim e, negativa se todas as respostas fossem não.

A presença de diabetes é avaliada com a pergunta: "nos últimos doze meses você teve diabetes?" Com as respostas sim ou não. 
A pergunta: "nos últimos doze meses, você passou a noite como paciente em algum hospital?" Com as respostas: nenhuma vez, uma vez, duas ou três vezes, e mais do que três vezes avalia-se o número de internações hospitalares.

A variável consulta médica nos últimos doze meses foi avaliada com a questão: "nos últimos doze meses, quantas vezes você procurou o médico ou uma clínica para tratamento?". Cabendo as seguintes respostas: nenhuma vez, uma vez, duas ou três vezes, quatro a seis vezes e mais de seis vezes.

\section{Digitação e Análise dos dados}

Os dados foram digitados utilizando-se o programa Epi Info 6.04. Foram obtidas as frequências para cada variável e estratificados de acordo com a existência de internação no último ano.

\section{Aspectos Éticos}

A pesquisa foi realizada por meio da busca de dados nos prontuários dos usuários do Posto de Saúde de Bela União. O projeto foi encaminhado ao Comitê Científico da FUMSSAR.

\section{Resultados}

Foram avaliados 176 idosos com idade igual ou superior a 60 anos residentes em área rural do município de Santa Rosa (RS). Os idosos eram cadastrados no distrito de saúde de Bela União.

A Tabela 1 resume os principais resultados da pesquisa.

A distribuição por sexo mostra $57,4 \%$ da população estudada composta por mulheres e de $42,6 \%$ composta por homens.

Na análise da idade, encontrou-se a seguinte distribuição: $30,9 \%$ entre $60-64$ anos de idade; $30,3 \%$ entre 65 a 69 anos; $18,3 \%$ entre $70-74$ anos; $20,6 \%$ entre 75 anos ou mais. As faixas etárias diferem do trabalho de $\operatorname{Veras}^{10}[10]$, que analisou dados de idosos acima de 65 anos. No entanto, sugere neste mesmo estudo que faixas etárias mais jovens sejam incluidas em outros estudos.

Quanto à presença de doenças crônicas, $8,5 \%$ dos analisados possuía diabetes e $14,2 \%$ doença cardiaca. $O$ apoio social, neste trabalho definido como a presença de pessoa próxima a que possa recorrer caso precise, foi mencionado por $97,7 \%$ dos idosos. Quanto à autopercepçào de saúde, $5,7 \%$ considera a sua saúde excelente/muito boa; $25,6 \%$ boa; $63,6 \%$ média, e $5,1 \%$ ruim.

O número de consultas médicas nos últimos 12 meses anteriores à entrevista teve a seguinte distribuição: $18,8 \%$ não consultaram; $52,3 \%$ consultaram 1 a 3 vezes, e $29 \%$ o fizeram mais de 3 vezes. Foram agrupadas as variáveis para quem se consultou 2 ou 3 vezes, 4- 6 vezes, e mais de 6 vezes, ficando essas englobadas em uma só: mais de três vezes.

Realizou-se comparação das demais variáveis com o fato de ter internado nos últimos 12 meses. Observou-se uma tendência de internação com pior autopercepção da saúde. O maior número de consultas no último ano e a presença de doença cardíaca foram fatores estatísticamente associados à internação. A presença de diabetes e de apoio social tiveram a análise prejudicada pelo pequeno número de indivíduos em um dos grupos, mas parecem indicar para uma associação, enquanto sexo e idade não apresentaram associação com internação nos últimos 12 meses.

\section{Discussão}

A saúde do idoso não deve ser medida somente pela presença ou ausência de patologias, e sim pela autonomia apresentada pelo idoso frente às suas limitaçòes. Os fatores que determinam o envelhecimento saudável e as incapacidades resultantes de uma ou mais doenças crônicas devem ser alvo de estudos, pois conhecer a populaçào idosa é imperativo no planejamento das ações de saúde oferecidas.

O predomínio de mulheres idosas $(57,4 \%)$ encontrado no estudo segue a tendència mundial onde se observa uma feminização do envelhecimento.

Ao analisarmos a morbidade, encontramos um percentual de $8,5 \%$ de idosos com diabetes e $14,2 \% \mathrm{com}$ doença cardíaca. Na cidade onde se realizou o estudo, $34,1 \%$ 
do total de óbitos em 2004 devem-se a essas patologias. Índices menores aos observados, por exemplo, nos estudos $\mathrm{SABE}^{12}$ onde foram estudados idosos com 60 anos ou mais, residentes em áreas urbanas de sete países da América
Latina e Caribe, incluindo o município de São Paulo (SP), e encontrou-se um percentual de 17,9\% para diabetes e 14,2\% de doença cardíaca; no estudo de Veras ${ }^{10}$ com percentual de $25 \%$ de diabetes e $35,1 \%$ de doença cardíaca na popula-

Tabela 1. Distribuição dos fatores de risco para internações repetidas entre a população de 60 anos ou mais em unidade de saúde rural do Município de Santa Rosa - RS

\begin{tabular}{|c|c|c|}
\hline Características & Frequência n & Percentual \% \\
\hline Autopercepção da Saúde & & 18 \\
\hline Excelente / muito boa & 10 & 5,7 \\
\hline Boa & 45 & 25,6 \\
\hline Média & 112 & 63,6 \\
\hline Ruim & 9 & 5,1 \\
\hline \multicolumn{3}{|l|}{ Hospitalização } \\
\hline Nenhuma vez & 151 & 85,8 \\
\hline $1 \mathrm{vez}$ & 20 & 11,4 \\
\hline 2 ou mais vezes & 5 & 2,8 \\
\hline \multicolumn{3}{|l|}{ Consultas Médicas } \\
\hline Nenhuma vez & 33 & 18,8 \\
\hline 1 a 3 vezes & 92 & 52,3 \\
\hline mais de 3 vezes & 51 & 29,0 \\
\hline \multicolumn{3}{|l|}{ Diabetes Mellitus } \\
\hline Sim & 15 & 8,5 \\
\hline Não & 161 & 91,5 \\
\hline \multicolumn{3}{|l|}{ Doença Cardiovascular } \\
\hline Sim & 25 & 14,2 \\
\hline Não & 151 & 85,8 \\
\hline \multicolumn{3}{|l|}{ Sexo } \\
\hline Feminino & 101 & 57,4 \\
\hline Masculino & 75 & 42,6 \\
\hline \multicolumn{3}{|l|}{ Apoio Social } \\
\hline Sim & 172 & 97,7 \\
\hline Não & 4 & 2,3 \\
\hline \multicolumn{3}{|l|}{ Idade } \\
\hline $60-64$ anos & 54 & 30,9 \\
\hline $65-69$ anos & 53 & 30,3 \\
\hline $70-74$ anos & 32 & 18,3 \\
\hline 75 anos ou mais & 36 & 20,6 \\
\hline Total & 176 & 100,0 \\
\hline
\end{tabular}


ção idosa com mais de 65 anos de idade e residentes em área urbana. A baixa prevalência de diabetes e doença cardíaca no estudo pode estar relacionada ao menor sedentarismo da população idosa na área rural, sendo a atividade física uma das intervenções preventivas mais efetivas na prevenção dessas patologias ${ }^{2,13}$.
A autopercepção da saúde tem se mostrado um indicador consistente e robusto na avaliação global da saúde do idosos. Indivíduos que consideram a sua saúde ruim têm risco de mortalidade aumentada ${ }^{12}$, demonstrando que a autopercepçào da saúde pode ser tão acurada quanto outros determinantes objetivos da saúde. Os resultados

Tabela 1. Distribuição das variáveis de acordo com a presença ou não de internações entre a população de 60 anos ou mais em unidade de saúde rural - Santa Rosa - RS.

\begin{tabular}{|c|c|c|c|c|c|}
\hline \multirow[t]{3}{*}{ Exposições } & \multicolumn{5}{|c|}{ Internações nos últimos 12 meses } \\
\hline & \multicolumn{2}{|c|}{ Sim } & \multicolumn{3}{|c|}{ Não } \\
\hline & $\mathrm{n}$ & $\%$ & $\mathrm{n}$ & $\%$ & $\mathrm{P}$ \\
\hline \multicolumn{6}{|l|}{ Autopercepção da Saúde } \\
\hline Excelente / muito boa & 2 & 20,0 & 8 & 80,0 & 0,25 \\
\hline Boa & 4 & 8,9 & 41 & 91,1 & \\
\hline Média & 16 & 14,3 & 96 & 85,7 & \\
\hline Ruim & 3 & 33,3 & 6 & 66,7 & \\
\hline \multicolumn{6}{|l|}{ Sexo } \\
\hline Feminino & 13 & 12,9 & 88 & 87,1 & 0,71 \\
\hline Masculino & 12 & 16,0 & 63 & 84,0 & \\
\hline \multicolumn{6}{|l|}{ Consultas Médicas } \\
\hline Nenhuma vez & 0 & 0,0 & 33 & 100,0 & 0,01 \\
\hline 1 a 3 vezes & 13 & 14,1 & 79 & 85,6 & \\
\hline mais de 3 vezes & 12 & 23,5 & 39 & 76,5 & \\
\hline \multicolumn{6}{|l|}{ Idade } \\
\hline $60-64$ anos & 7 & 13,0 & 47 & 87,0 & 0,90 \\
\hline $65-69$ anos & 7 & 13,2 & 46 & 86,8 & \\
\hline $70-74$ anos & 5 & 15,6 & 27 & 84,4 & \\
\hline 75 anos ou mais & 6 & 16,7 & 30 & 83,3 & \\
\hline \multicolumn{6}{|l|}{ Diabetes Mellitus } \\
\hline Nào & 22 & 13,7 & 139 & 86,3 & 0,77 \\
\hline $\operatorname{Sim}$ & 3 & 20,0 & 12 & 80,0 & \\
\hline \multicolumn{6}{|l|}{ Doença Cardiovascular } \\
\hline Não & 18 & 11,9 & 133 & 88,1 & 0,04 \\
\hline Sim & 7 & 28,0 & 18 & 72,0 & \\
\hline \multicolumn{6}{|l|}{ Apoio Social } \\
\hline $\operatorname{Sim}$ & 25 & 14,5 & 147 & 85,5 & * \\
\hline Não & 0 & 0,0 & 4 & 100,0 & \\
\hline
\end{tabular}

* em toda a amostra apenas 4 pessoas não identificaram apoio social 
encontrados no estudo realizado em Santa Rosa, demonstram que os idosos têm uma boa percepção de sua saúde, $63,6 \%$ a consideraram média contrastando com $5,1 \%$ que a consideram ruim. Quando estratificada por sexo, verificamos que as mulheres possuem uma pior autopercepção da saúde quando comparadas aos homens $(60 \%$ do sexo masculino a consideram excelente/muito boa em comparação com 40\% entre as mulheres - dados não apresentados). No estudo de Veras ${ }^{10}$, realizado no Rio de Janeiro e com uma população-alvo composta de pessoas com 65 anos ou mais, os valores são semelhantes.

A análise evidenciou um número elevado de idosos realizando mais de três consultas por ano, contudo, quase $1 / 5$ não realizou consulta médica. Destaca-se a importância do atendimento ao idoso. Os que realizaram mais consultas foram os que mais internaram. O conhecimento da utilização do serviço é importante para as equipes de ESF na organização de suas ofertas de serviço, já que nestes a demanda por consultas ultrapassa a oferta, sendo necessária a utilização de critérios de prioridade e avaliação de risco na organizaçâo da demanda ${ }^{14}$. A associação entre internação e maior número de consultas pode evidenciar a presença de morbidade e/ou pior estado de saúde, reforçando a importância do atendimento na atenção primária aos que internaram, como oportunidade de realizar ações que previnam novas internações. Cabe lembrar que a presença de internação hospitalar relaciona-se com perda da autonomia e capacidade fundional do idoso ${ }^{3}$ e que a proporção de pessoas acima de 70 anos internada é quase o triplo quando comparado a outras faixas etárias ${ }^{15}$.

A grande maioria dos indivíduos estudados possuía apoio familiar $(97,7 \%)$ corroborando com os achados de $\mathrm{Negri}^{16} \mathrm{e} \mathrm{Veras}^{10} \mathrm{em}$ estudos semelhantes. O efeito protetor das relaçôes sociais sobre diversos aspectos da saúde do idoso, desde seu padrão alimentar até a mortalidade, tem sido reconhecido. As características do apoio social devem ser consideradas pelas ESF que prestam atendimento aos idosos.

\section{Conclusão}

O trabalho realizado possibilitou conhecer, por meio da descrição dos fatores de risco para internações repetidas, a população de idosos residentes na área de abrangência de ESF no município de Santa Rosa (RS).

A escolha da população estudada foi determinada por ser área de atuação de um dos autores e reforça a necessidade de mais estudos abrangendo a população de área rural, pois, em estudos semelhantes, a população estudada é de idosos residentes em área urbana ${ }^{910}$. A maioria dos estudos sobre o envelhecimento populacional referese aos determinantes da transição epidemiológica, avaliação dos serviços de saúde, sendo as informações sobre a saúde do idoso e os seus determinantes escassos ${ }^{4}$.

A análise da distribuição de fatores de risco para internações repetidas em idosos fornece dados para uma adequação das ações em saúde oferecidas ao idoso. O cuidado ao idoso na atenção primária deve basear-se na estratégia de Equipes de Saúde da Família com profissionais especialistas na área. Essas equipes estão capacitadas para compreender o processo de envelhecimento identificando os fatores que determinam a qualidade de vida do idoso e atuar de maneira a mantê-lo o mais independente possíve] no desempenho de suas atividades ${ }^{8}$.

Por fim, destacamos a importância das ESF inseridas em área rural envolverem-se em investigações científicas sobre a saúde do idoso, por tratar-se de indivíduos com piores índices de saúde devido a vários fatores, dentre eles o baixo grau de escolaridade, apresentando, portanto, maior vulnerabilidade. $\mathrm{E}$ ainda, devido à escassa existência de estudos abrangendo a população idosa residente em área rural.

\section{Referências}

1. BRasil. Ministério da Saúde. Política Nacional do Idoso. Portaria GM 1965/99 ed: Diário Oficial 1999:20-4.

2. Chaimowicz F. A Saúde dos idosos brasileiros às vésperas do século XXI: problemas, projeçōes e alternativas. Rev. Saúde Pública. 1997 1997;31(2):184-200.

3. Ramos LR. Fatores determinantes do envelhecimento 
saudável em idosos residentes em centro urbano: Projeto Epidoso, São Paulo. Cadernos de Saúde Pública. 2003 2003;19(3):796-8.

4. Rouquayrol MZ, Filho N. Epidemiologia e Saúde. 6 ed. Rio de Janeiro: Guanabara Koogan; 2003.

5. IBGE F. Acesso e utilização dos serviços de saúde. In: Pesquisa Nacional por Amostras de Domicilio. [s.l.: s.n.];: 2005.

6. Veras RP, Ramos LR, Kalache A. Crescimento da população idosa no Brasil: transformações e consequências na sociedade. Rev Saúde Pública. Jun 1987 ; 21(3):225-33.

7. Benvegnú LA, Moroni U, Chitolina T. Boletim Epidemiológico FUMSSAR. Santa Rosa; 2005.

8. Silvestre JA, da Costa Neto MM. Abordagem do idoso em programas de saúde da família. Cad. Saúde Pública. Maio-Jun. 2003;19(3):839-47.

9. Guerra IC, Ramos-Cerqueira AT. Risco de hospitalizações repetidas em idosos usuários de um centro de saúde escola. 2007; 23(3):585-92.[falta o tótilo do periódico]

10. Veras R. Em busca de uma assistência adequada à saúde do idoso: revisão da literatura e aplicação de um instrumento de detecção precoce e de previsibilidade de agravos. Cad. Saúde Pública. Maio-Jun. 2003; 19(3):705-15.

11. Alves LC, Rodrigues RN. Determinantes da Autopercepção de Saúde entre Idosos do município de São Paulo, Brasil. Revista Panamericana de Salud Pública. 2005;17(5/6):333-41.

12. Lebrão ML, Laurenti R. Saúde, bem-estar e envelhecimento: o estudo SABE no Município de São Paulo. Rev Bras Epidemiol. 2005; 8(2):127-41.

13. Duncan BB, Schmidt MI, Giugliani E. Medicina Ambulatorial: condutas de Atenção Primária Baseadas em Evidências. 3 ed. Porto Alegre ArtMed; 2004.

14. Lourenco RA, Martins Cde S, Sanchez MA, Veras RP. [Geriatric outpatient healthcare: hierarchical demand structuring.]. Rev. Saúde Pública. Apr .2005; 39 (2):311-8.

15. Chaimowicz F, Ferreira LG, Martins TJX, Assumpção DF. Uso de medicamentos psicoativos e seu relacionamento com quedas entre idosos. Rev. Saúde Pública. 2000; 34(6). 16. Negri LSA, Ruy GF, Colodetti JB, Pinto LF, Soranz DR. Aplicação de um instrumento para detecção precoce e previsibilidade de agravos na população idosa. Ciència \& Saúde Coletiva. 2004; 9(4):1033-46.

\section{Endereço para Correspondência:}

Rua Doralino Leusin, 126.

Santa Rosa - RS.

CEP: $98.900-000$.

\section{Endereço eletrônico:}

fabianabreitenbach@brturbo.com.br 